\title{
Uso das Tecnologias de Informação e Comunicação no Curso de Medicina da UFRN
}

\author{
Use of Information and Communication \\ Technologies in the UFRN Medicine Course
}

\author{
Jocekleyton Ramalho da Silva \\ Fabiano Barros de Medeiros ${ }^{I}$ \\ Frankswell Mackson Soares de Moura ${ }^{I}$ \\ Wellington da Silva Bessa ${ }^{I}$ \\ Elaine Lira Medeiros Bezerra ${ }^{I}$
}

PALAVRAS-CHAVE

- Tecnologia;

- Educação Médica;

- Ensino;

- Rede Social.

\section{KEYWORDS}

- Technology;

- Medical Education;

- Teaching;

- Social Networking.
Recebido em: 06/10/2014

Aprovado em: 08/08/2015

\section{RESUMO}

Está claro na literatura que as Tecnologias de Informação e Comunicação (TICs) oferecem um ambiente acadêmico virtual que melhora a interação entre docentes e alunos. Baseado nisto, nosso trabalho descreveu de que modo o estudante de Medicina da Universidade Federal do Rio Grande do Norte utiliza as TICs. Também investigou quais dessas ferramentas são utilizadas e a interferência da lingua inglesa emseu uso. Esta pesquisa éum estudo descritivo de delineamento transversal, cujo instrumento de coleta de dados foi um questionário autoaplicável, padronizado e anônimo. Ao final do estudo, obteve-se uma taxa geral de respostas de $82 \%(n=489)$. Verificou-se que as redes sociais são utilizadas por quase a totalidade dos estudantes (97,3\%), que há um uso mais frequente de artigos científicos para o aprendizado ao longo dos períodos e que o uso majoritário da língua inglesa nessas ferramentas não constitui uma barreira para a maior parte da amostra (72\%).

ABSTRACT
It is clear in the literature that Information and Communication Technologies (ICTs) offer a virtual
academic environment that enhances interaction between teachers and students. Based on this, our
work described how medical studentsat theFederal University of Rio Grande do Norteuse ICTs. The
investigation focused on which of these tools are used and the interference of the English language
in the use of ICTs.This research is a descriptive, cross-sectional study, which drew on data collected
through a self-administered,standardized and anonymous questionnaire.Bythe end of the study we
obtained an overall response rate of $82 \%$ ( $n=489$ ). It was found that social networks are used by al-
most all the students (97.3\%); it was also observed that there is more frequent use of scientific articles
for learning throughout academic terms and that the use of the English language by the majority of
ICTs does not constitute a barrier for most of the sample (72\%).

\section{ABSTRACT}

It is clear in the literature that Information and Communication Technologies (ICTs) offer a virtual academic environment that enhances interaction between teachers and students. Based on this, our work described how medical studentsat theFederal University of Rio Grande do Norteuse ICTs. The investigation focused on which of these tools are used and the interference of the English language in the use of ICTs. This research is a descriptive, cross-sectional study, which drew on data collected through a self-administered,standardized and anonymous questionnaire.Bythe end of the study we obtained an overall response rate of $82 \%(n=489)$. It was found that social networks are used by almost all the students (97.3\%); it was also observed that there is more frequent use of scientific articles ICTs does not constitute a barrier for most of the sample (72\%). 


\section{INTRODUÇÃO}

Nos últimos anos, a tecnologia reorganizou a forma como vivemos, nos comunicamos e aprendemos. Antes, a vigência do conhecimento era medida em décadas; hoje, com os avanços das Tecnologias da Informação e Comunicação (TICs), o conhecimento está crescendo exponencialmente, sendo atualizado em intervalo de tempo bem menor ${ }^{1}$.

Na área médica, alternativas de acesso à informação estão sendo adaptadas às inovações tecnológicas como forma de tentar suprir esse grande acúmulo de informações. É o caso do E-learning, termo que traduz o uso da internet e de outras ferramentas, assim como diversas bases de dados, informações científicas e programas computacionais destinados a melhorar o conhecimento e o desempenho estudantil ${ }^{2,3}$.

Diversos estudos concluíram que as TICs contribuem para o aprendizado em várias áreas da Medicina, como Anatomia ${ }^{4}$, Pediatria $^{5,6}$, Patologia ${ }^{7}$ e Oncologia ${ }^{8}$, entre outras. Esses trabalhos utilizaram uma variedade de TICs em ambientes acadêmicos nos quais o conhecimento dos alunos era medido pelas pontuações pré e pós-teste; constatou-se que os estudantes que utilizaram essas tecnologias aprenderam de forma mais eficiente e demonstraram melhor retenção de conhecimento. Além disso, uma meta-análise de estudos comparando o ensino baseado em TICs com os métodos tradicionais de ensino revelou uma melhora estatisticamente significantecom o uso dessas ferramentas 9 .

As Diretrizes Curriculares Nacionais (DCN) para os cursos de graduação em Medicina também abordam o tema,estabelecendo como uma competência geral do médico o domínio das TICs, assim como a habilidade de decidir a conduta mais apropriada com base em evidências científicas e a competência da educação continuada,a qual está diretamente ligada à capacidade do médico para utilizar as $\mathrm{TICs}^{10}$.

Além disso, diante da globalização, a quantidade de informações veiculadas pelas TICs, além de aumentar, passou a ser disponibilizada preferencialmente em língua inglesa - língua dominante no campo da ciência,em que as pesquisas em saúde estão predominantemente inseridas. Isto justifica a evidência de que não saber inglês é um obstáculo à busca de informações para o desenvolvimento de pesquisa científica e conhecimento no contexto atual, o que pode ser um diferencial no desempenho cultural de um estudante ${ }^{11}$. As DCN também reconhecem a importância do domínio de uma língua estrangeira, citando essa necessidade como uma competência geral do médico ${ }^{10}$.

As Instituições de Ensino Superior (IES) também têm buscado formas de otimizar o ensino e o gerenciamento de suas informações, recorrendo a sistemas tecnológicos de informação acadêmica. A Universidade Federal do Rio Grande do
Norte (UFRN) possui o Sistema Integrado de Gestão de Atividades Acadêmicas (Sigaa), que, além de auxiliar nos processos acadêmicos de matrícula e cadastro em atividades científicas, entre outras, também contribui para o ensino além do limite físico da sala de aula por criar um ambiente de contato virtual entre docentes e discentes ${ }^{12}$.

Contudo, a tecnologia, por si só, não garante uma educação de qualidade e de sucesso ${ }^{13}$. Qualquer tecnologia só é válida em educação quando estudantes e professores fazem com ela algo de útil, ou seja, quando por meio dela e com ela experimentam situações de aprendizagem significativa, construindo conhecimento ${ }^{14,15}$

Diante do exposto, conhecer como as TICs são utilizadas pelos estudantes de Medicina motivou a realização deste estudo, que tem como objetivos descrever de que modo o estudante do curso de Medicina da UFRN utiliza as TICs para seu aprendizado, assim como avaliar se a língua inglesa interfere no uso dessas tecnologias.

\section{METODOLOGIA}

Foi realizado um estudo descritivo e transversal no período de julho a novembro de 2013. Foram incluídos no estudo todos os alunos matriculados no curso de Medicina da UFRN, sendo utilizado como instrumento de coleta de dados um questionário autoaplicável, padronizado e anônimo. Os alunos foram informados sobre os objetivos do estudo, e aqueles que aceitaram assinar o Termo de Consentimento Livre e Esclarecido (TCLE) preencheram um questionário com informações sobre período no curso, idade, sexo, renda familiar, equipamentos eletrônicos que possuem, frequência de uso de redes sociais, interferências da língua inglesa no uso das TICs e com que frequência utilizam bases de artigos científicos, UpToDate (um sistema de banco de dados de informação médica) e Sigaa para o aprendizado.

Os dados foram analisados por meio do SPSS (software utilizado para análises estatísticas) versão 21.0. Para facilitar a visualização dos dados, os estudantes foram agrupados em três grupos de acordo como período que estavam cursando: do primeiro ao quarto (componentes curriculares predominantemente básicos), do quinto ao oitavo (componentes curriculares predominantemente clínicos) e do nono ao décimo segundo período (internato). Para verificar a relação entre o período no curso e a frequência de utilização de bases on-line, Sigaa e UpToDate, foi o utilizado o teste chi-quadrado de Pearson, adotando-se como significativos valores de $\mathrm{p}<0,005$. O estudo foi realizado de acordo com a Resolução 196/96 do Conselho Nacional de Saúde e foi aprovado pelo Comitê de Ética em Pesquisa de Seres Humanos da Universidade Federal do Rio Grande do Norte, sob o Parecer nº 369.459. 


\section{RESULTADOS}

Do total dos 598 alunos regularmente matriculados no curso de Medicina da UFRN no período letivo 2013.2, 489 (82\%) responderam ao questionário, sendo $250(51,1 \%)$ do sexo masculino e $239(48,9 \%)$ do sexo feminino, com a média geral de idade de 22,8 anos. Em relação à renda salarial, 81,4\%dos alunos possuem renda acima de quatro salários mínimos. Todos os entrevistados têm pelo menos um dos seguintes equipamentos eletrônicos: computador desktop, notebook, smartphone, tablet (Tabela 1).

\begin{tabular}{|c|c|c|}
\hline \multicolumn{3}{|c|}{ TABELA 1} \\
\hline & $\mathrm{n}$ & $\%$ \\
\hline \multicolumn{3}{|l|}{ Sexo } \\
\hline Feminino & 239 & 48,9 \\
\hline Masculino & 250 & 51,1 \\
\hline \multicolumn{3}{|l|}{ Idade (média) } \\
\hline Feminino & 22,5 & \\
\hline Masculino & 23,2 & \\
\hline \multicolumn{3}{|c|}{ Renda (em salários mínimos) } \\
\hline Até 2 & 23 & 4,7 \\
\hline 2 a 4 & 70 & 14,3 \\
\hline 4 a 8 & 129 & 26,4 \\
\hline$>8$ & 267 & 54,6 \\
\hline \multicolumn{3}{|c|}{ Equipamentos eletrônicos } \\
\hline Computador desktop & 215 & 44,0 \\
\hline Notebook & 447 & 91,4 \\
\hline Smartphone & 382 & 78,1 \\
\hline Tablet & 230 & 47,0 \\
\hline
\end{tabular}

As redes sociais são usadas por 476 (97,3\%) estudantes, sendo que $277(62 \%)$ as utilizam mais de três vezes ao dia (Tabela 2). Em relação ao uso de TICs em língua inglesa, 352 (72\%) afirmam que isto não constitui uma barreira para seu uso. A Tabela 3 detalha a frequência com que os estudantes utilizam as seguintes ferramentas para seu aprendizado: bases on-line, UpToDate e Sigaa.

\section{TABELA 2}

Frequência da utilização de redes sociais pelos estudantes de Medicina na UFRN

\begin{tabular}{|c|c|c|}
\hline Frequência & $\mathbf{n}$ & $\%$ \\
\hline Nunca & 13 & 2,7 \\
\hline Nem todos os dias & 46 & 9,4 \\
\hline $1-3 x /$ dia & 152 & 31,1 \\
\hline $4-6 x /$ dia & 134 & 27,4 \\
\hline$>6 x /$ dia & 144 & 29,4 \\
\hline
\end{tabular}

\begin{tabular}{|c|c|c|c|c|c|c|c|c|c|c|c|}
\hline $\begin{array}{l}\text { Distr } \\
\text { aprer }\end{array}$ & $\begin{array}{l}\text { ibu } \\
\text { diz }\end{array}$ & $\begin{array}{l}\text { ão } \\
\text { do }\end{array}$ & $\begin{array}{l}\text { da } \mathrm{f} \\
\text { pelo }\end{array}$ & $\begin{array}{r}\mathrm{T} \\
\mathrm{equ} \\
\mathrm{alu}\end{array}$ & t & do & us & in & da & $\mathbf{p}$ & \\
\hline Frequência/ & & & $\begin{array}{l}\text { Nen } \\
\text { ser }\end{array}$ & $\begin{array}{l}\text { toda } \\
\text { ana }\end{array}$ & & & & & & & $\mathrm{p}$ \\
\hline & $\mathrm{n}$ & $\%$ & $\mathrm{n}$ & $\%$ & $\mathrm{n}$ & $\%$ & n & $\%$ & $\mathrm{n}$ & $\%$ & \\
\hline Artigo científi & & & & & & & & & & & $<0,005$ \\
\hline $1^{\mathrm{o}}-4^{\mathrm{o}}$ & 27 & 16 & 123 & 74 & 13 & 8 & 3 & 2 & 0 & 0 & \\
\hline $5^{\circ}-8^{o}$ & 7 & 4 & 118 & 69 & 29 & 17 & 14 & 8 & 3 & 2 & \\
\hline $9^{\circ}-12^{\mathrm{o}}$ & 0 & 0 & 11 & 7 & 65 & 43 & 65 & 43 & 11 & 7 & \\
\hline UpToDate & & & & & & & & & & & $<0,005$ \\
\hline $1^{\circ}-4^{o}$ & 98 & 59 & 50 & 30 & 13 & 8 & 2 & 1 & 3 & 2 & \\
\hline $5^{\circ}-8^{\circ}$ & 19 & 11 & 101 & 59 & 31 & 18 & 12 & 7 & 9 & 5 & \\
\hline $9^{\circ}-12^{\mathrm{o}}$ & 2 & 1 & 6 & 4 & 46 & 30 & 65 & 43 & 33 & 22 & \\
\hline Sigaa & & & & & & & & & & & $<0,005$ \\
\hline $1^{\circ}-4^{o}$ & 18 & 11 & 27 & 16 & 68 & 41 & 33 & 20 & 22 & 13 & \\
\hline $5^{\circ}-8^{o}$ & 15 & 9 & 101 & 59 & 36 & 21 & 14 & 8 & 7 & 4 & \\
\hline $9^{\circ}-12^{\circ}$ & 36 & 24 & 47 & 31 & 41 & 27 & 20 & 13 & 8 & 5 & \\
\hline
\end{tabular}

\section{DISCUSSÃO}

Observou-se que há um uso muito baixo de bases de dados on-line pelos estudantes de Medicina da UFRN do primeiro ao quarto período, momento em que predominam disciplinas do ciclo básico, como Anatomia, Fisiologia, Histologia, Embriologia. Entretanto, essas bases tornam-se bastante utilizadas a partir do nono período, quando se inicia o internato médico, e o aluno passa a ter a vivência mais prática da medicina, havendo também maior necessidade de obtenção de evidências científicas que embasem uma conduta clínica mais atualizada.

Esse achado contrasta com o de outros estudos, como um realizado numa universidade da Finlândia ${ }^{16}$ que evidenciou que os estudantes de Medicina apresentam alto nível de utilização de bases de dados científicos desde o primeiro ano do curso, fato este impulsionado por disciplinas como Informática Médica e Bioestatística, presentes no início do curso. Disciplinas dessa natureza também estão presentes nocurrículo do curso de Medicina da UFRN, porém não são obrigatórias.

No que se refere ao uso do UpToDate, observou-se que $75,7 \%$ dos estudantes o utilizam. Esse valor foi superior ao encontrado por Peterson et al. ${ }^{17}$ num estudo na Universidade de Iowa (EUA) em que 53\% dos alunos relataram utilizar o UpToDate como fonte preferencial de informações. Entre os fatos que podem justificar o uso considerável desta ferramenta está o acesso gratuito oferecido aos acadêmicos da UFRN, assim como seu design prático, que permite uma navegação fácil e simples.

Segundo estudo ${ }^{12}$ realizado também na UFRN, o Sigaa contribuiu para melhor interação aluno-professor, ajudou na aprendizagem ao fornecer um contato mais fácil e rápido, e permitiu ao professor uma forma mais interativa de disseminar materiais que possam ajudar o aluno, o que melhorou o 
cotidiano de discentes e docentes no que diz respeito à busca e oferta de conhecimento por meio da comunicação. Ao analisar sua utilização, observou-se um uso considerável desse sistema $-86 \%$ dos estudantes -, achado também encontrado por outros estudos ${ }^{18}$.

Entretanto, foi observada uma diferença significativa com relação à frequência de acesso ao Sigaa entre os períodos, sendo maior no início do curso. Esse achado alerta para uma subutilização desse sistema, que poderia gerar um ambiente virtual de fácil comunicação entre docente e discente, permitindo, por exemplo, formar grupos para discutir casos clínicos, disponibilizar diretrizes atualizadas, esclarecer dúvidas que não puderam ser respondidas em sala de aula, entre outras funcionalidades.

No que diz respeito ao uso de redes sociais, encontramos que seu uso é bastante frequente, uma vez que 97,3\% dos entrevistados as utilizam, sendo essa uma tendência encontrada também por outros estudos ${ }^{18}$. Esse dado aponta a utilização das redes sociais como um novo ambiente de ensino, uma vez que elas permitem criar um ambiente colaborativo e de construção do conhecimento ${ }^{19,20}$.

Em relação à influência da língua inglesa no uso das TICs, um estudo ${ }^{11}$ demonstrou que, em 2003, 97\% das indexações no Institute for Scientific Information (ISI) estavam em inglês, assim como, avaliando-se a base de dados Medline, identificou-se que 84\% das publicações em revistas médicas em 2005 utilizavam a língua inglesa. As $\mathrm{DCN}^{10}$ também fazem referência à importância do domínio de uma língua estrangeira ao colocarem essa característica como uma competência do médico. Nesse contexto, a maioria dos estudantes de Medicina da UFRN (72\%) considerou não ter dificuldade em utilizar TICs baseadas nessa língua.

Um aspecto metodológico importante foi a escolha de aplicar questionários impressos, uma vez que estudos que utilizaram questionários enviados por e-mail obtiveram uma taxa de resposta inferior à alcançada em nosso estudo ${ }^{16}$. Essa diferença no índice de respostas nos leva a questionar se o grande montante de informações que podem ser transmitidas através de uma TIC - como, por exemplo, os e-mails que recebemos todos os dias - pode ser superior a nossa capacidade de processamento de informações, nos levando a negligenciar parte delas, o que explicaria a menor taxa de resposta aos questionários enviados por $e$-mail ${ }^{21}$.

\section{CONCLUSÃO}

Diante dos resultados deste estudo, é possível concluir que a maioria dos alunos do curso de Medicina da UFRN tem condições de acesso às TICs, tanto em hardware como em software, e faz destas um meio de estudo prevalente. Pode-se concluir também que bases on-line, Up To Date e Sigaa são ferramentas fortemente utilizadas no meio acadêmico. Ao observar o aumento da intensidade de uso desses meios de informação ao longo dos períodos, pode-se inferir que isto ocorre em virtude do grande contingente de informações disponíveis e que são atualizadas a todo instante, tornando-se mais necessárias ao longo do curso.

Finalmente, não se pode deixar de observar que a língua inglesa, dominada pela maioria dos estudantes pesquisados, está intrinsecamente relacionada a maior liberdade de acesso e manipulação das diversas formas de TICs.

\section{REFERÊNCIAS}

1. Hay M, Canny B. Effective use of information and communications technology in distributed medical assessment. MedEduc [online].2011. 45(11) [capturado 28 jan. 2014]; 1154-1155. Disponível em: http://onlinelibrary.wiley. com/doi/10.1111/j.1365-2923.2011.04114.x/abstract;jsessi onid=FF7E431859B92B009CDE0141A4713077.f02t02.

2. Norman G. Effectiveness, efficiency, and e-learning. Adv Health SciEduc [online].2008. 13(3) [capturado 28 jan. 2014]; 249-251.Disponível em: http:/ /link.springer.com/ article/10.1007\%2Fs10459-008-9131-5.

3. Ruiz JG, Mintzer MJ, Leipzig RM. The Impact of E-Learning in Medical Education. AcadMed [online].2006. 81(3) [capturado 28 jan. 2014]; 207-212. Disponível em: http:/ / www.ncbi.nlm.nih.gov/pubmed/16501260.

4. Nicholson DT1, Chalk C, Funnell WR, Daniel SJ. Can virtual reality improve anatomy education? A randomised controlled study of a computer generated three dimensional anatomical ear model.MedEduc [online].2006. 40(11) [capturado 15 fev. 2014]; 1081-1087. Disponível em: http:// www.ncbi.nlm.nih.gov/pubmed/17054617.

5. O'Leary FM, Janson P. Can e-learning improve medical students knowledge and competence in pediatric cardiopulmonary resuscitation? A prospective before and after study.Emerg Med [online].2010. 22(4) [capturado 15 fev. 2014]; 324-329.Disponível em: http:/ / www.ncbi.nlm.nih. gov/pubmed/20629697.

6. Ricks C, Ratnapalan S, Jain S, Tait G. Evaluating computer-assisted learning forcommon pediatric emergency procedures. Pediatr. Emerg. Care [online].2008. 24(5) [capturado 15 fev. 2014]; 284-286. Disponível em: http://www.ncbi. nlm.nih.gov/pubmed/18496110.

7. Velan GM, Lattimore M, Lindeman R, Kumar RK. A web-based module onlymphoma for senior medical students: benefits for learning.JIAMSE [online].2010. 20(1) [captura- 
do 15 fev. 2014]; 32-40. Disponível em: http:/ / www.iamse. org/artman/publish/article_540.shtml.

8. Morgulis Y, Kumar RK, Lindeman R, Velan GM. Impact on learning of na e-learning module on leukaemia: a randomised controlled trial. BMC MedEduc[online].2012. 12(5) [capturado 16 mar. 2014]; 36-45. Disponível em: http:// www.biomedcentral.com/content/pdf/1472-6920-12-36. pdf.

9. Cook DA, Levinson AJ, Garside S, Dupras DM, Erwin PJ, Montori VM: Internet-based learning in the health professions. JAMA 2008;300(10)1181-1196.

10. Brasil. Conselho Nacional de Educação. Câmara de Educação Superior. Resolução CNE/CES nº 116/2014 de 20 de junho de 2014. Institui diretrizes curriculares nacionais do curso de graduação em Medicina. Diário Oficial da União. Brasília, 6 jun. 2014; Seção 1, p.17.

11. Regina S, Iglesias A, Batista NA. A língua inglesa e a formação de mestres e doutores na área da saúde. Rev. bras. educ. med. 2010; 34(1):74-81.

12. Queiroz FCBP, Hekis HR, Queiroz JV, Oliveira LAB, Vasconcelos NVC. Contribuição dos Sistemas Integrados de Gestão para as Práticas de Ensino e Aprendizagem. Augusto Guzzo Revista Acadêmica 2012; 9(1):45-52.

13. Lifschitz V, Bobadilla A, Esquivel P, Giusiano G, Merino L. Aplicación Del aprendizajebasadoen problemas para La enseñanza de La microbiologia em estudiantes de Medicina. Educ. méd 2010; 13(2):107-111;

14. Schlinkert WR, Scarpelini S, Pazin-filho A. Problems and potentialities of e-Learning for regular undergraduate courses in emergency medicine. Rev. bras. educ. med. 2010;34(3):452-458.

15. Gomez MV, VieiralJE, Neto AS. Análise do Perfil de Professores da Área da Saúde que Usam a Simulação como Estratégia Didática. Rev. bras. educ. med 2011;35(2):157-162.

16. Romanov K, Aarnio M. A survey of the use of electronic scientific information resources among medical and dental students. BMCMedEduc[online].2006. 6(1) [capturado 02 mar. 2014]; 28-36. Disponível em:http://www.ncbi.nlm. nih.gov/pmc/articles/PMC1479814/.

17. Peterson MW, Rowat J, Kreiter C, Mandel J. Medical students' use of information resources: Is the digital age dawning. AcadMed [online].2004. 79(1) [capturado 03 mar.
2014]; 89-95. Disponível em: http://www.ncbi.nlm.nih. gov/pubmed/14691004.

18. Câmara FMP, Oliveira DFM, Silva RA, Paulo VT, Abreu EPF, Neto CM, et al. Perfil de manuseio das inovações tecnológicas pelo estudante de medicina e sua interface para o aprendizado em saúde. R-BITS [online].2014. 4(1) [capturado 10 jul. 2014]; 52-60. Disponível em: http:/ /www. periodicos.ufrn.br/reb/article/view / 4549.

19. Estus EL. Using Facebook within a geriatric pharmacotherapy course. Am J Pharm Educ. [online].2010. 74(8) [capturado 07 jun. 2014]; 1-5. Disponível em: http:/ / www.ncbi. nlm.nih.gov/pmc/articles/PMC2987285/.

20. DiVall MV, Kirwin JL. Using Facebook to facilitate course-related discussion between students and faculty members. Am J Pharm Educ. [online].2012. 76(2) [capturado 07 jun. 2014];1-5. Disponível em: http://www.ncbi.nlm.nih. gov/pubmed/22438604.

21. Thomas WJ, Pourya F. Theory-based model of factors affecting information overload. International Journal of Information Management [online].2012. 32(6) [capturado 01 out. 2014]; 523-532. Disponívelem: http://www.sciencedirect.com/science/article/pii/S02684012 12000606.

\section{CONTRIBUIÇÃO DOS AUTORES}

Declaramos que todos os autores participaram de forma suficiente da concepção e desenho deste estudo, da análise e da interpretação dos dados, assim como da redação deste texto.

\section{CONFLITO DE INTERESSES}

Não houve conflito de interesses durante a elaboração deste estudo.

\section{ENDEREÇO PARA CORRESPONDÊNCIA}

Jocekleyton Ramalho da Silva

Universidade Federal do Rio Grande do Norte

Av. Bernardo Vieira, 4114- Lagoa Nova - Natal

CEP 59056-045 - RN

E-mail:jocekleyton@hotmail.com 